\title{
About half permutations
}

\author{
Simone Rinaldi Samanta Socci \\ Dipartimento di Ingegneria dell'Informazione e Scienze Matematiche \\ University of Sienna \\ Italy \\ rinaldi@unisi.it, socci5@unisi.it
}

Submitted: Jun 20, 2013; Accepted: Feb 16, 2014; Published: Feb 21, 2014

Mathematics Subject Classifications: 05A05, 05A15

\begin{abstract}
In 2011, Beaton et al. analytically proved that the number of directed columnconvex permutominoes of size $n$ is given by $(n+1) ! / 2$. In this paper, we provide a different proof of this statement using a bijective method. More precisely, we present a bijective correspondence between the class $\mathcal{D}_{n}$ of directed column-convex permutominoes of size $n$ and a set of permutations (called $d c c$-permutations) of length $n+1$, which we prove to be counted by $(n+1) ! / 2$. The class of $d c c$-permutations is a new class of permutations counted by half factorial numbers, and here we show some combinatorial characterizations of this class, using the concept of logical formulas determined by a permutation and the notion of mesh pattern.
\end{abstract}

\section{Introduction}

Let $P$ be a polyomino without "holes", i.e. a polyomino whose boundary is a single loop, and having $n-1$ rows and $n-1$ columns, $n \geqslant 2$; we assume without loss of generality that the south-west corner of its minimal bounding rectangle is placed at $(1,1)$. We recall that $P$ is a permutomino if for each abscissa (ordinate) between 1 and $n$ there is exactly one vertical (horizontal) bond in the boundary of $P$ with that coordinate, and $n-1$ is called the size of the permutomino. A permutomino of size $n-1$ can be equivalently defined by a pair $\left(\pi_{1}(P), \pi_{2}(P)\right)$ of permutations of length $n$ (see Figure 1 ). These permutations are called the first and the second component of $P$, respectively. We refer to $[3,9,11]$ for more detailed definitions on polyominoes and on permutominoes, and to [6] for basic definitions on permutations.

Permutominoes were introduced in [13] in some algebraic context and then they were considered by F. Incitti, in the study of the $\widetilde{R}$-polynomials associated with a pair $\left(\pi_{1}, \pi_{2}\right)$ of permutations [12]. The most significant results on the enumeration of column-convex 


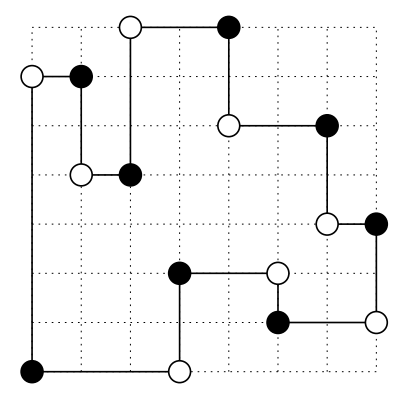

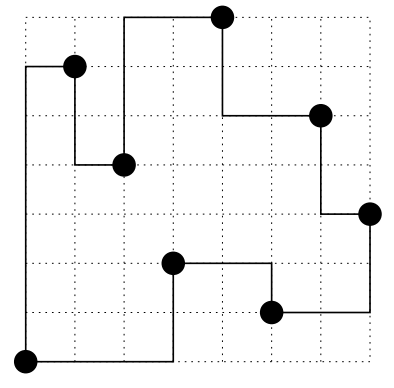

$\pi_{1}=(1,7,5,3,8,2,6,4)$

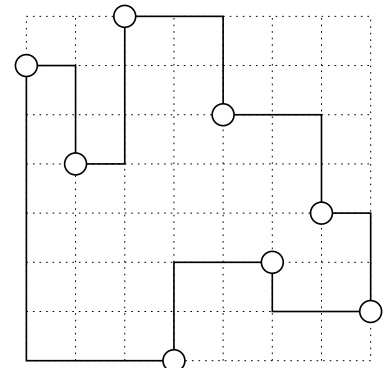

$\pi_{2}=(7,5,8,1,6,3,4,2)$

Figure 1: A permutomino of size 7 and the two associated permutations.

permutominoes was obtained in [2], where the authors determined a direct recursive construction for the column-convex permutominoes of a given size, leading to a functional equation. However they were not able to solve the equation in order to obtain the generating function of column-convex permutominoes. By using numerical analysis they were led to conjecture that the number $f_{n}$ of column-convex permutominoes of size $n$ has the following asymptotic behavior:

$$
f_{n} \sim k(n+1) ! h^{n}
$$

where $k=0.3419111$ and $h=1.385933$. Exact results have been obtained for some classes of convex permutominoes $[3,9,11]$, and in particular the number of convex permutominoes of size $n$ was proved to be equal to:

$$
2(n+3) 4^{n-2}-\frac{n}{2}\left(\begin{array}{c}
2 n \\
n
\end{array}\right) \quad n \geqslant 1 .
$$

We point out that (1) was proved independently in [5] and in [9], by using analytical techniques. Recently, a bijective proof of (1) was given in [8] by encoding convex permutominoes in terms of lattice paths.

Permutominoes have recently been studied in some different research areas, such as discrete and computational geometry: in fact they have been considered by Tomas and Bajuelos under the name of grid n-ogons [1]. Later Eppstein [10] introduced the concept of $x y z$-graph, which is any 3-regular graph which can be embedded into 3-dimensional Cartesian coordinates, in such a way that two vertices are adjacent if and only if two of their three coordinates lie on an axis-parallel line and such that no three points lie on the same axis-parallel line. Eppstein pointed out that $x y z$-graphs can be considered as a generalization of permutominoes to 3 -dimensions.

In this paper we consider the class $\mathcal{D}_{n}$ of directed column-convex permutominoes of size $n \geqslant 1$. We recall that a polyomino is said to be column-convex if all its columns are connected, and north-east directed (briefly, directed) if all its cells can be reached from a distinguished cell - called the source - by means of a path, internal to the polyomino, 
which uses only north and east unit steps (see Figure 2(a)). The authors of [2] analytically proved that the number of directed column-convex permutominoes of size $n$ is $(n+1) ! / 2$.

At the recent conference GASCom 2012, held in Bordeaux, we have been asked for a direct combinatorial explanation of this enumerative result. The simpler proof of this fact would consist in a bijective correspondence between $\mathcal{D}_{n}$ and one of the several known occurrences of classes permutations counted by $(n+1) ! / 2$. Unfortunately, we have not been able to find one such a "natural" correspondence, so we were motivated to look for a more complex proof.

In this paper, we reach this goal by proving that:

- every directed column-convex permutomino $P$ is uniquely determined by its second component $\pi_{2}(P)$

- the set $\mathcal{P}_{n+1}^{\prime \prime}=\left\{\pi_{2}(P): P \in \mathcal{D}_{n}\right\}$ is in bijective correspondence with the set $S_{n+1} \backslash \mathcal{P}_{n+1}^{\prime \prime}$, where $S_{n}$ denotes, as usual, the set of permutations of length $n$.

We point out that the objects of $\mathcal{P}_{n}^{\prime \prime}$, called dcc-permutations, are a nontrivial set of permutations counted by half the factorial numbers, and they have not been considered yet in the literature. So, in the second part of the paper we study some combinatorial properties of this set. In particular, we succeed in giving an alternative characterization of dcc-permutations, using the concept of logical formulas determined by a permutation, recently introduced in [4]. Thank to this result, we are finally able to characterize the set $d c c$-permutations as a class of permutations avoiding two mesh patterns [7].

\section{Enumeration of directed column-convex permut- ominoes}

Throughout the paper $S_{n}$ will denote the set of permutations of length $n, \mathcal{D}_{n}$ the set of directed column-convex permutominoes of size $n$, and $\mathcal{P}_{n}^{\prime}$ (resp. $\mathcal{P}_{n}^{\prime \prime}$ ) the set of first (resp. second) components of permutominoes in $\mathcal{D}_{n-1}$. Clearly, $\mathcal{P}_{n}^{\prime}, \mathcal{P}_{n}^{\prime \prime} \subseteq S_{n}$.

Given $\pi=(\pi(1), \ldots, \pi(n)) \in S_{n}$, we will use the graphical representation of $\pi$ made with the points $(i, \pi(i))$, and, by abuse of notation, we will often refer to the point $\pi(i)$ of $\pi$, to mean the point $(i, \pi(i))$. If $n>1, \pi(1) \neq 1$, and $j$ is the index such that $\pi(j)=1$, let $\mathcal{R}(\pi)$ (briefly, $\mathcal{R}$ ) be the array of right-to-left minima of $\pi$ and $\overline{\mathcal{R}}(\pi)$ (briefly, $\overline{\mathcal{R}}$ ) be the array of the entries $(\pi(j-1), \pi(j), \ldots, \pi(n))$ of $\pi$ minus the points which are right-to-left minima of $\pi$. The rightmost element of $\overline{\mathcal{R}}(\pi)$ will be referred to as $L(\pi)$ (briefly, $L$ ). So, for instance, referring to $\pi=(6,12,8,1,11,2,3,5,9,7,10,4)$ represented in Figure 2(a), we have $\mathcal{R}=(1,2,3,4), \overline{\mathcal{R}}=(8,11,5,9,7,10)$, and $L=10$.

We start encoding a directed column-convex permutomino of size $n-1$ by means of a permutation of length $n$.

Proposition 1. A directed column-convex permutomino $P$ is uniquely determined by its second component $\pi_{2}(P)$. 
Proof. The proof follows from the observation that the permutation $\pi_{2}(P)$ (briefly, $\pi_{2}$ ) forces the vertices of $P$ to be connected in a unique way. More precisely (see Figure 2(a)):

a) $\pi_{2}(1)$ is connected with $\pi_{2}(i)=1$, because $P$ is a directed polyomino;

b) the entries of $\pi_{2}$ which are right-lo-left minima - i.e., the elements of $\mathcal{R}\left(\pi_{2}\right)$ - have to be connected in sequence because $P$ is directed. In the graphical representation we will always depict the path connecting the right-to-left minima by means of a dashed line;

c) there is a unique way to connect the remaining entries of $\pi_{2}$, since $P$ is a column-convex permutomino.

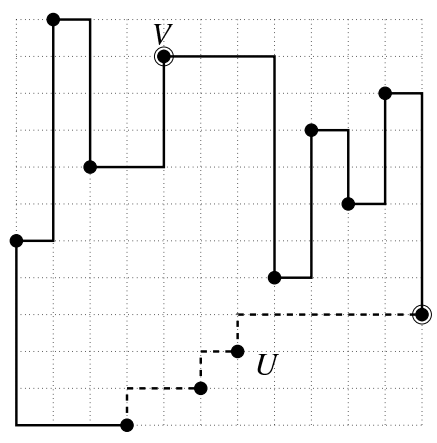

(a)

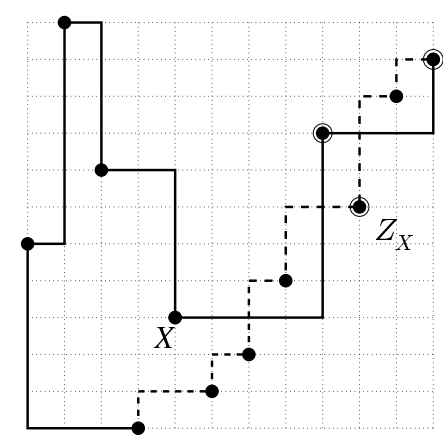

(b)

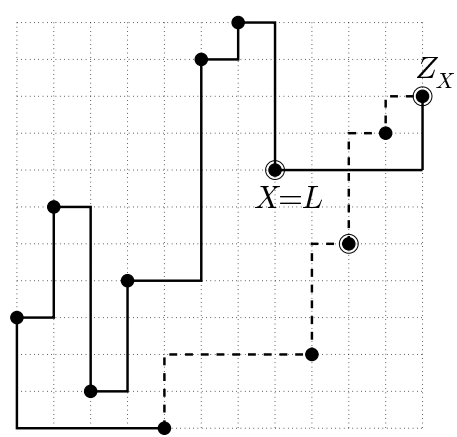

(c)

Figure 2: (a) A directed column-convex permutomino, and its second component $\pi_{2} \in \mathcal{P}_{n}^{\prime \prime}$; (b) The permutation corresponding to $\pi_{2}$ through the mapping $\phi^{-1}$; (c) A permutation that does not satisfy condition $i i i$ ).

The permutations of $\mathcal{P}_{n}^{\prime \prime}$ will be then called directed column-convex permutations (briefly, dcc-permutations).

Definition 2. Let $\pi \in S_{n}$ such that $\pi(1) \neq 1$; for each $X \in \overline{\mathcal{R}}-\{L\}$, let $Y$ be the leftmost point of $\overline{\mathcal{R}}$ on the right of $X$, and $Z$ the leftmost point of $\mathcal{R}$ on the right of $Y$. We set $Z_{X}=Z$.

Figure 2(b) depicts a permutation, the point $X=4$, and the point $Z_{X}=7$. Observe that possibly $Z_{X}=\pi(n)$.

Theorem 3. A permutation $\pi$ of length $n$ is a dcc-permutation if and only if the following properties hold:

i) $\pi(1) \neq 1$;

ii) for each point $X \in \overline{\mathcal{R}}(\pi)-\{L\}$, we have $X>Z_{X}$;

iii) $L>\pi(n)$. 
Proof. We start observing that the three properties are independent; in particular, condition $i i i$ ) can be regarded as a degenerate case of condition $i i$ ) where $X=L$ and $Z_{X}=\pi(n)$. In any case, our proof turns out to be simpler if we keep conditions $i i$ ) and $i i i$ ) separated.

Let $P$ be a directed column-convex permutomino. We have already observed that the elements of the set $\mathcal{R}\left(\pi_{2}(P)\right)$ identify the lower path of $P$ and the remaining elements of $\pi_{2}(P)$ identify the other part of the boundary of $P$ (the upper path). It is clear that $\pi_{2}(P)$ must satisfy $\left.\left.i\right), i i\right)$, and $\left.i i i\right)$, since the violation of any of the three conditions would cause the two sub-paths to cross themselves. Figure 2(b) shows a permutation not satisfying condition $i$ ), while Figure 2(c) shows a permutation satisfying $i i$ ) but not $i i i$ ). The reader can check that in both cases it is not possible to construct a directed columnconvex permutomino such that $\pi_{2}(P)=\pi$, then $\pi$ is not a $d c c$-permutation.

Conversely, let $\pi$ be a permutation satisfying $i$ ), $i i$ ) and $i i i)$. In this case, we can build the corresponding directed column-convex permutomino $P$ according to the rules $a$ ), $b$ ) and $c$ ) in Proposition 1.

We just need to prove that the upper path and the lower path cannot cross themselves. In practice, provided $\pi(1) \neq 1$, Figure 3 shows that if the upper path and the lower path cross themselves, then we run across the violation of one or both conditions ii) or iii). Now, by construction, we have $\pi_{2}(P)=\pi$, then $\pi$ is a $d c c$-permutation.

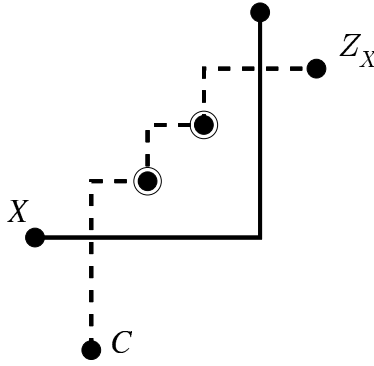

(a)

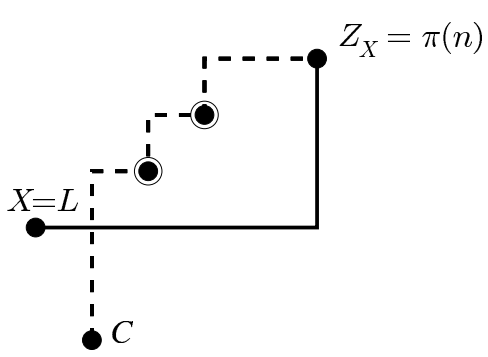

(b)

Figure 3: The two possible ways that the upper and the lower path may cross themselves; the encircled points need not be present, while there is necessarily a right-to-left minimum $C$ of $\pi$, since otherwise $X$ itself would be a right-to-left minimum.

Our aim is now to determine the number of $d c c$-permutations of length $n$, which by Proposition 1 - is equal to the number of directed column-convex permutominoes of size $n-1$.

Theorem 4. For $n \geqslant 2$, the number of dcc-permutations of length $n$ is $\frac{n !}{2}$.

Proof. To prove this statement, we determine a bijective correspondence

$$
\phi: S_{n} \backslash \mathcal{P}_{n}^{\prime \prime} \rightarrow \mathcal{P}_{n}^{\prime \prime}
$$

So, let $\pi \in S_{n} \backslash \mathcal{P}_{n}^{\prime \prime}$. We distinguish three cases, depending on whether $\pi$ violates condition $i$ ), ii) (and not $i$ ) or $i i i$ ) (and not $i$ ), ii)): 
(1) $\pi(1)=1$. In this case, $\phi$ maps $\pi=(1, \pi(2) \ldots \pi(n))$ onto the permutation $\pi^{\prime}=$ $(\pi(2) \ldots \pi(n), 1)$. Clearly, since $\pi^{\prime}(n)=1, \pi^{\prime}$ satisfies properties $\left.\left.i\right), i i\right)$ and $\left.i i i\right)$, so $\pi^{\prime} \in \mathcal{P}_{n}^{\prime \prime}$.

(2) $\pi$ satisfies $i$ ) but not $i$ ). In this case there is at least an element $X \in \overline{\mathcal{R}}-\{L\}$ such that $X<Z_{X}$, as depicted in Figure 2(b). So, let $X^{\prime}$ be the leftmost of these elements (clearly $X^{\prime} \neq 1$ ) and set $\phi(\pi)$ to be equal to the permutation obtained from $\pi$ by exchanging $X^{\prime}$ with $\pi(n)$ (see Figure $2(\mathrm{a})$ ).

(3) $\pi$ satisfies $i$ ) and $i i$ ) but not $i i i$ ), i.e. $L<\pi(n)$ (as in Figure 4(b)). In this case $\phi(\pi)$ is obtained from $\pi$, by exchanging $L$ with $\pi(n)$. Figure 4(a) shows the permutation obtained from that in Figure 4(b).

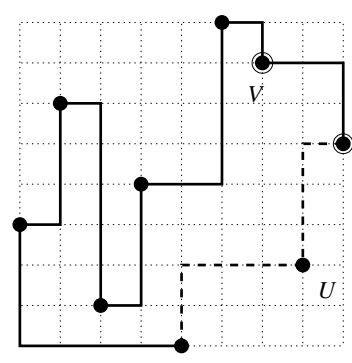

(a)

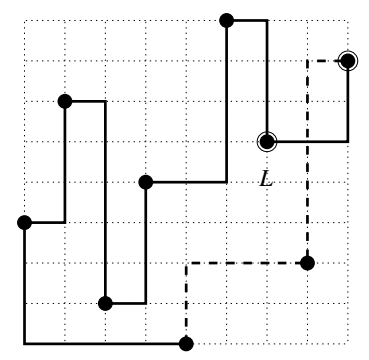

(b)

Figure 4: (a) A directed column-convex permutomino, and the associated permutation $\pi_{2}(P) \in \mathcal{P}_{n}^{\prime \prime}$; (b) The permutation $\pi=\phi^{-1}\left(\pi_{2}\right)$, where $L(\pi)<\pi(n)$ (condition iii) is not satisfied).

Now we prove that the obtained permutation $\pi^{\prime}=\phi(\pi)$ is a $d c c$-permutation, i.e. it satisfies conditions $i$ ), $i i$ ), and $i i i$ ) of Theorem 3 .

First, we assume that $\pi$ falls in case (2). We observe that the set of right-to-left minima of $\pi^{\prime}=\phi(\pi)$ is precisely

$$
\mathcal{R}\left(\pi^{\prime}\right)=\left\{P: P \text { is a right-to-left minimum of } \pi \text { and } P<X^{\prime}\right\} \cup\left\{X^{\prime}\right\},
$$

and that all points in $\overline{\mathcal{R}}\left(\pi^{\prime}\right)$ lying on the right of $\pi(n)$ are greater than all points of $\mathcal{R}\left(\pi^{\prime}\right)$, thus $\pi^{\prime}$ satisfies $i i$ ), and $i i i$ ). Case (3) is analogous to the previous one, it suffices to replace $X^{\prime}$ by $L$.

Finally, we need to prove that $\phi$ is a bijection. This is trivial if $\pi^{\prime}(n)=1$, since we fall in case (1). Otherwise, we can invert the function $\phi$ as follows: let us consider a $d c c-$ permutation $\pi^{\prime}=\phi(\pi)=\left(\pi^{\prime}(1), \ldots, \pi^{\prime}(n)\right)$; let $U$ be the rightmost right-to-left minima of $\pi^{\prime}$ different from $\pi^{\prime}(n)$, and $V$ the rightmost element in $\overline{\mathcal{R}}\left(\pi^{\prime}\right)$ on the left of $U$. The points $U$ and $V$ have been marked in Figure 2(a) and Figure 4(a). By exchanging $V$ with $\pi^{\prime}(n)$ we obtain the permutation $\pi^{\prime \prime}$.

By construction $\pi^{\prime \prime}$ is not a $d c c$-permutation; more precisely we can prove that $\pi^{\prime \prime}=\pi$, since: 
- if $\pi^{\prime \prime}$ does not satisfy $\left.i i\right)$, then $\pi^{\prime}(n)$ is just the leftmost element $X \in \overline{\mathcal{R}}\left(\pi^{\prime \prime}\right)-\{L\}$ such that $X<Z_{X}$;

- if $\pi^{\prime \prime}$ satisfies $i i$ ) but not $\left.i i i\right)$, then $\pi^{\prime}(n)$ is the rightmost element $X$ which is not a right-to-left minima (i.e. $L$ ).

It follows that $\phi$ is a bijection.

\section{Combinatorial characterizations of $d c c$-permutations}

Theorem 4 tells us that the set of $d c c$-permutations is a new class of permutations counted by half factorial numbers. Despite several efforts, we have not been able to relate such a class with any of the known classes counted by these numbers. This is why we believe it interesting to determine some combinatorial properties, and some alternative characterizations of $d c c$-permutations.

Let us recall that a permutation $\pi$ is called decomposable if there is an index $i<n$ such that $(\pi(1), \ldots, \pi(i))$ is a permutation. Similarly, $\pi$ is $R$-decomposable if its reverse $\pi^{r}$ is decomposable.

We also need to recall some basic concepts and definitions from [4], where the authors introduced the notion of logical implications associated with a permutation, and used these implications to characterize the set of the first components of column-convex permutominoes. Let $P$ be a column-convex permutomino of size $n$, let $\pi_{1}(P)$ (briefly, $\pi_{1}$ ) be the first component of $P$, and let $U_{i}=\left(i, \pi_{1}(i)\right), 1 \leqslant i \leqslant n+1$, be the points of the graphical representation of $\pi_{1}$. As in the previous section, we call upper (resp. lower) path of $P$ the part of the boundary of $P$ running from $U_{1}$ to $U_{n+1}$ and starting with a north step (resp. east step). It follows from the definition that the upper path remains strictly above the lower path except at the points $U_{1}$ and $U_{n+1}$. To any point $U_{i}=\left(i, \pi_{1}(i)\right)$ we associate the value

$$
v\left(U_{i}\right)= \begin{cases}1 & \text { if } U_{i} \text { belongs to the upper path } \\ 0 & \text { otherwise }\end{cases}
$$

The points $U_{1}$ and $U_{n+1}$ belong both to the upper and the lower paths, and, for our convenience, we set $v\left(U_{1}\right)=0$, and $v\left(U_{n+1}\right)=1$. Clearly, a column-convex permutomino $P$ is uniquely determined by $\pi_{1}(P)$, and by the array $v\left(\pi_{1}\right)=\left(v\left(U_{1}\right), \ldots, v\left(U_{n+1}\right)\right)$. For instance, the column-convex permutomino $P$ in Figure $5(\mathrm{a})$ is uniquely determined by its first component $\pi_{1}(P)=(2,6,3,5,1,7,4)$ and by the valuation $v\left(\pi_{1}\right)=(0,1,0,1,0,1,1)$.

Definition 5. Let $U_{i}, U_{j} \in \mathcal{U}$, we say that the pair $\left(U_{i}, U_{j}\right)$ forms an inversion if and only is $i<j$ and $\pi(i)>\pi(j)$. Moreover, we say that the array $\left[U_{i}, U_{j}\right]=\left(U_{i}, U_{i+1}, \ldots, U_{j}\right)$ is a locally decomposable ( $R$-decomposable) permutation if the normalization of

$$
(\pi(i), \pi(i+1), \ldots, \pi(j))
$$

is a decomposable ( $R$-decomposable) permutation. 


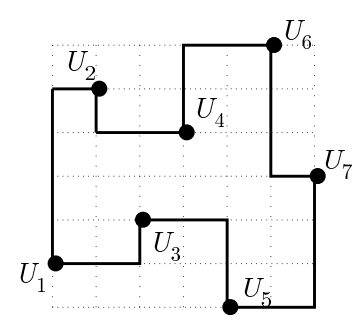

(a)

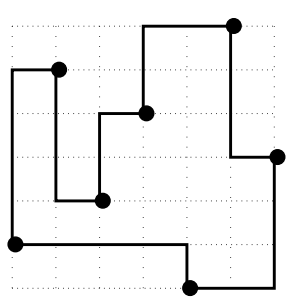

(b)

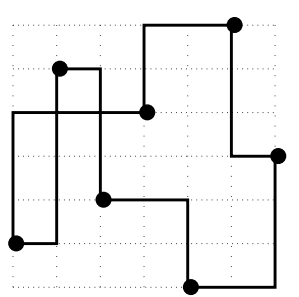

(c)

Figure 5: Two column convex permutominoes having $\pi=(2,6,3,5,1,7,4)$ as their first component: (a) non north-west directed; (b) north-west directed; (c) a valuation which does not satisfy $\mathcal{F}(\pi)$.

For any given $\pi \in S_{n}$ we define a set of logic implication formulas $\mathcal{F}(\pi)$ on the variables $\mathcal{U}=\left\{U_{1}, \ldots, U_{n}\right\}$. We point out that each variable uniquely refers to a point of $\pi$, and from now on, we will use indifferently the symbol $U_{i}$ for a point or for the corresponding variable.

Definition 6. Given $U_{i}, U_{j} \in \mathcal{U}$ we set $U_{j} \rightarrow U_{i} \in \mathcal{F}(\pi)$ if $\left(U_{i}, U_{j}\right)$ is an inversion and the array $\left[U_{i}, U_{j}\right]$ is locally R-decomposable.

Given a valuation $v$, and two points $A, B$, the valuation of $A \rightarrow B$ is defined as follows:

$$
v(A \rightarrow B)=0 \text { if and only if } v(A)=1 \text { and } v(B)=0 .
$$

The reader may observe that this is just the standard valuation of the implication $A \rightarrow B$ adopted in propositional calculus. If $v(A \rightarrow B)=1$ we say that $v$ satisfies $A \rightarrow B$. Now, we say that $v$ satisfies a set of implications $\mathcal{F}$ if it satisfies all the implications of $\mathcal{F}$. We say that $\mathcal{F}(\pi)$ is satisfiable if there is at least a valuation $v$ of $\pi$ which satisfies all the formulas of $\mathcal{F}(\pi)$.

So, for instance, referring to $\pi=(2,6,3,5,1,7,4)$, depicted in Figure $5(\mathrm{a})$, we have that $\mathcal{F}(\pi)=\left\{U_{3} \rightarrow U_{2}, U_{4} \rightarrow U_{2}, U_{5} \rightarrow U_{1} U_{2} U_{3} U_{4}, U_{7} \rightarrow U_{6}\right\}$. The reader can check that the valuations $v_{1}(\pi)=(0,1,0,1,0,1,1)$, and $v_{2}=(0,1,1,1,0,1,1)$ satisfy $\mathcal{F}(\pi)$ (in fact they correspond to the permutominoes in Figure $5(\mathrm{a}),(\mathrm{b})$, respectively), whereas the valuation $v_{3}(\pi)=(0,0,0,1,0,1,1)$ does not satisfy $\mathcal{F}(\pi)$; in fact, as depicted in Figure 4(c), if we build the the upper and lower paths according to $v_{3}$, we have that paths cross themselves.

In [4] the authors proved that the set of permutations

$$
\mathcal{C}^{\prime}(n)=\left\{\pi_{1}(P): P \text { is a column-convex permutomino of size } n\right\}
$$

can be characterized in terms of the satisfiability of the associated set of implications.

Theorem 7. A permutation $\pi \in \mathcal{C}^{\prime}(n)$ if and only if $\mathcal{F}(\pi)$ is satisfiable. 
We can use the concept of logical formulas associated with a permutation, and in particular Theorem 7 , in order to provide an alternative characterization of $d c c$-permutations. Before proceeding we just need to observe that, given a permutomino $P$, the first component of $P$ is just the reverse of the second component of the polyomino $P^{M}$ obtained by reflecting $P$ with respect to the $y$-axis. Moreover, a permutomino $P$ is north-east directed column-convex if and only if $P^{M}$ is north-west directed column-convex.

Definition 8. Given a permutation $\pi$, let $\hat{v}$ be the valuation of $\pi$ defined as follows:

$$
\hat{v}\left(U_{i}\right)=0 \text { if and only if } U_{i} \text { is a left-to-right minimum. }
$$

Using Proposition 1 and Theorem 7 we can state:

Proposition 9. A permutation $\pi$ of length $n$ is a dcc-permutation if and only if the valuation $\hat{v}$ satisfies $\mathcal{F}\left(\pi^{r}\right)$.

Figure 5(b) depicts a north-west directed column-convex permutomino $P^{M}$, whose first component is the permutation $\pi=(2,6,3,5,1,7,4)$. We can see that the valuation $\hat{v}=(0,1,1,1,0,1,1)$ satisfies $\mathcal{F}(\pi)$, hence $\pi^{r}$ is a $d c c$-permutation. Clearly, the (unique) north-east directed permutomino having $\pi^{r}$ as its second component is just $P$, obtained by reflecting $P^{M}$ with respect to the $y$-axis. Now we are ready to state our main result:

Theorem 10. A permutation $\pi$ of length $n$ is a dcc-permutation if and only if:

i) $\pi(1) \neq 1$,

ii) $\mathcal{F}\left(\pi^{r}\right)$ is satisfiable,

iii) for every implication $U_{i} \rightarrow U_{1}$ belonging to $\mathcal{F}\left(\pi^{r}\right)$, we have that $U_{i}$ is a left-to-right minimum.

Proof. $(\Rightarrow)$ Let us assume that one of the three conditions is not satisfied:

i) if $\pi(1)=1$ then clearly $\pi$ is not a $d c c$-permutation;

ii) if $\mathcal{F}\left(\pi^{r}\right)$ is not satisfiable then $\pi^{r} \notin \mathcal{C}^{\prime}(n)$ then $\pi$ is not a $d c c$-permutation;

iii) if there is an implication $U_{i} \rightarrow U_{1}$ belonging to $\mathcal{F}\left(\pi^{r}\right)$ where $U_{i}$ is not a left-toright minimum, then we have that, for all $v$ satisfying $\mathcal{F}\left(\pi^{r}\right), v\left(U_{i}\right)=0$. Then the valuation $\hat{v}$ does not satisfy $\mathcal{F}\left(\pi^{r}\right)$, hence, by Proposition $9, \pi$ is not a $d c c$ permutation.

$(\Leftarrow)$ By Proposition 9 it is sufficient to prove that $\hat{v}$ satisfies $\mathcal{F}\left(\pi^{r}\right)$. Let us assume by contradiction that it does not hold; then there is an implication $U_{j} \rightarrow U_{i} \in \mathcal{F}\left(\pi^{r}\right)$ such that $\hat{v}\left(U_{j} \rightarrow U_{i}\right)=0$, i.e. $\hat{v}\left(U_{j}\right)=1$ (i.e., $U_{j}$ is not a left-to-right minimum) and $\hat{v}\left(U_{i}\right)=0$ (i.e., $U_{i}$ is a left-to-right minimum). Since $U_{i}$ is a left-to-right minimum, by Definition 6 , it follows that $U_{i} \rightarrow U_{1} \in \mathcal{F}\left(\pi^{r}\right)$. Then, by transitivity we have $U_{j} \rightarrow U_{1} \in \mathcal{F}\left(\pi^{r}\right)$, which contradicts hypothesis iii), since $U_{j}$ is not a left-to-right minimum. 
The three conditions of Theorem 10 can be re-formulated in a more suitable way.

Corollary 11. A permutation $\pi$ of length $n$ belongs to $\mathcal{P}_{n}^{\prime \prime}$ if and only if $\pi(1) \neq 1$ and there is not any point $U_{i}$ of $\pi$ such that $\left[U_{i}, U_{n}\right]$ is a locally decomposable permutation and $U_{i}$ is not a right-to-left minimum.

The previous result can be used to provide a characterization of $d c c$-permutations in terms of mesh patterns. We recall that mesh patterns are a generalization of bi-vincular patterns in permutations, recently introduced in [7]. In a word, a mesh pattern $(\pi, R)$ is an occurrence of the permutation pattern $\pi$ with additional restrictions specified by $R$ on the relative position of the entries of the permutation (see Figure 6). These restrictions can be suitably represented by means of shaded regions in the graphical representation of the pattern, and say that no elements of the permutation are allowed in the shaded regions.

Theorem 12. A permutation $\pi$ is a dcc-permutation if and only if $\pi$ avoids the mesh patterns:

$$
\begin{aligned}
& M_{1}=(213,\{(1,2),(1,3),(2,0),(2,1),(3,0),(3,1),(3,2),(3,3)\}) \\
& M_{2}=(1,\{(0,0),(0,1),(1,0)\})
\end{aligned}
$$

represented below

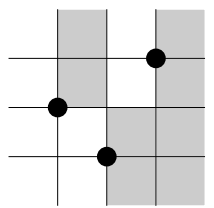

(a)

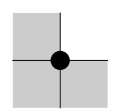

(b)

Figure 6: (a) The mesh pattern $M_{1}$; (b) The mesh pattern $M_{2}$.

Proof. $(\Rightarrow)$ Let us distinguish the following two cases:

(a) $\pi$ contains the pattern $M_{2}$. In practice, it means that $\pi(1)=1$, so $\pi$ is not a dcc-permutation;

(b) $\pi$ contains the pattern $M_{1}$. In this case, there are three entries $U_{i}, U_{j}, U_{k}$, with $i<j<k$, that form such mesh pattern. Let us consider the point $U_{i}$. Clearly, $U_{i}$ is not a right-to-left minimum of $\pi$, and $\left[U_{i}, U_{n}\right]$ is a locally decomposable permutation. Then, according to Corollary $11, \pi$ is not a $d c c$-permutation.

$(\Leftarrow)$ If $\pi$ is not a $d c c$-permutation, then, according to Corollary 11, two cases may occur:

(a) $\pi(1)=1$; in this case, $\pi$ contains the pattern $M_{2}$; 
(b) there is at least a point $U_{i}$ of $\pi$ such that is not a right-to-left minimum and $\left[U_{i}, U_{n}\right]$ is a locally decomposable permutation; so let $B$ be the rightmost point of these points, and let $A$ be the rightmost right-to-left minimum such that $A<B$ and let $C$ be the point $U_{n}$, then the three points $A, B$ and $C$ give rise to the mesh pattern $M_{1}$.

Referring to Figure 2(b),(c) the three points $A, B$ and $C$ which produce the pattern $M_{1}$ have been encircled.

At the end of our study, we have found it interesting to focus our attention on the class $\mathcal{B}_{n}$ of permutations avoiding the mesh pattern $M_{1}$; using the previous considerations, the enumeration of this class turns out to be straightforward.

Proposition 13. For $n \geqslant 2,\left|\mathcal{B}_{n}\right|=\sum_{i=0}^{n} \frac{i !}{2}$.

Proof. Let $\overline{\mathcal{B}}_{n}$ be the complement of $\mathcal{B}_{n}$, then $\left|\overline{\mathcal{B}}_{n}\right|=n$ ! $-\left|\mathcal{B}_{n}\right|$. Let $S_{n, 1}$ be the set of permutations of length $n$ starting with 1 , then $\left|S_{n, 1}\right|=(n-1)$ !. As a consequence of Theorem 4 and Theorem 12 have that:

$$
\frac{n !}{2}=n !-\left|\overline{\mathcal{B}}_{n}\right|-\left|S_{n, 1}\right|+\left|\overline{\mathcal{B}}_{n} \cap S_{n, 1}\right| .
$$

Now, we observe that $\overline{\mathcal{B}}_{n} \cap S_{n, 1}=\overline{\mathcal{B}}_{n-1}$, then we have:

$$
\left|\mathcal{B}_{n}\right|=\left|\mathcal{B}_{n-1}\right|+\frac{n !}{2}=1+\sum_{i=2}^{n} \frac{i !}{2}=\sum_{i=0}^{n} \frac{i !}{2} .
$$

\section{References}

[1] Bajuelos, A. L., Mafalda, A.M., Characterizing and Covering Some Subclasses of Orthogonal Polygons, Computational Science - ICCS 2006: 6th International Conference, LNCS 3992, Springer-Verlag (2006) 255-262.

[2] Beaton, N., Disanto, F., Guttmann, A.J., Rinaldi, S., On the enumeration of columnconvex permutominoes, 23th Formal Power Series and Algebraic Combinatorics, Proc. of Disc. Math. Theor. Comp. Sci., AO (2011) 111-122.

[3] Bernini, A., Disanto, F., Pinzani, R., Rinaldi, S., Permutations defining convex permutominoes, J. Int. Seq. 10 (2007) Article 07.9.7.

[4] Bilotta, S., Rinaldi, S., Socci, S., On polygons drawn from permutations, Fundamenta Informaticae 125 (2013) 329-342.

[5] Boldi, P., Lonati, V., Radicioni, R., Santini, M., The number of convex permutominoes, Inf. Comp. 206 (2008) 1074-1083.

[6] Bona, M., Combinatorics of permutations, Chapman-Hall and CRC Press (2004). 
[7] Brändén, P., Claesson, A., Mesh patterns and the expansion of permutation statistics as sums of permutation patterns, Electron. J. Combin. 18(2) P5.

[8] Disanto, F., Duchi, E., Pinzani, R., Rinaldi, S., Polyominoes determined by permutations: enumeration via bijections, Ann. Comb. 16 (2012) 57-75.

[9] Disanto, F., Frosini, A., Pinzani, R., Rinaldi, S., A closed formula for the number of convex permutominoes, El. J. Combinatorics 14 (2007) R57.

[10] Eppstein, D., The Topology of Bendless Three-Dimensional Orthogonal Graph Drawing, Graph Drawing, LNCS 5417 (2009) 78-89.

[11] Fanti, I., Frosini, A., Grazzini, E., Pinzani, R., Rinaldi, S., Characterization and enumeration of some classes of permutominoes, Pure Math. App., 18 No. 3-4 (2007) 265-290.

[12] Incitti, F., Permutation diagrams, fixed points and Kazdhan-Lusztig $R$-polynomials, Ann. Comb. 10 (2006) 369-387.

[13] Kassel, C., Lascoux, A., Reutenauer, C., The singular locus of a Schubert variety, J. Algebra 269 (2003) 74-108. 